\title{
Uniportal video-assisted thoracoscopic surgery in a two-year-old chronic empyema patient: challenges faced in the first case from the Indian subcontinent
}

\author{
Klein Dantis ${ }^{1}$, Habib Md Reazaul Karim², Mahesh S. Kurwe², Vikramjit Singh² \\ ${ }^{1}$ Department of Cardiothoracic and Vascular Surgery, All India Institute of Medical Sciences, Raipur, India \\ ${ }^{2}$ Department of Anaesthesiology and Critical Care, All India Institute of Medical Sciences, Raipur, India
}

Kardiochirurgia i Torakochirurgia Polska 2021; 18 (3): 177-179

Recently, there has been a rise in thoracic empyema in the pediatric population [1]. The uniportal video-assisted thoracoscopic surgery (U-VATS) for chronic empyema in adult patients has been advantageous as it is associated with less pain, faster recovery and better cosmetic results [2]. However, the role of U-VATS in pediatric patients below 3 years old is still anecdotal and less reported to date. Likewise, one-lung ventilation (OLV) in infants and young children is not unknown, but challenging, as the advantages of respiratory mechanics while positioned in lateral decubitus, as seen in adults, are absent in this age group [3]. Although OLV is described in children for video-assisted thoracoscopic surgery (VATS), experience and reports with U-VATS are scarce. We present a case of chronic empyema with lung abscess in a 2-year-old pediatric patient managed with U-VATS under general anaesthesia employing OLV.

A 2-year-old boy presented with dyspnea, fever, and cough for 4 weeks. On examination, he was tachypneic (35/ minute) with room air $\mathrm{SpO}_{2} 92 \%$, heart rate $145 /$ minute, and blood pressure $70 / 50 \mathrm{~mm} \mathrm{Hg}$. A chest X-ray showed consolidation with a loculated collection of the left lung (Figure 1). He was admitted to the intensive care unit (ICU) and started on empirical antibiotic treatment, which was changed later based on culture and sensitivity. Ultrasound chest was suggestive of the diffuse multiloculated collection; aspiration revealed minimal pus. However, fever and overall condition did not improve, and U-VATS decortication under general anaesthesia (GA) was planned.

With informed consent from the parent, the boy was taken under GA with OLV using single-use polyvinyl singlelumen, un-cuffed, an endotracheal tube of size $4 \mathrm{~mm} \mathrm{ID}$, intentionally progressed to the right endobronchial position. The tube position was confirmed using fibreoptic bronchoscopy. OLV was instituted and the patient positioned on the right lateral side. The diaphragm was marked according to the ultrasonographic appearance of a subpulmonic area before the incision.
A $2.5 \mathrm{~cm}$ muscle splitting incision was placed on the $5^{\text {th }}$ intercostal space between the mid-axillary and posterioraxillary line. A pediatric wound protector was inserted, followed by a $5 \mathrm{~mm} 30^{\circ}$ camera at the posterior aspect of the incision. Intraoperatively, an abscess over the superior segment of the left lower lobe was detected incidentally. A harmonic device was used to rupture the loculi, debridement was performed, and adhesions were lysed. Residual debris was removed using a forester sponge holding forceps. Parietal and visceral pleurectomy was done, and the lung was mobilized completely. Lung parenchyma was tested for air leak, followed by a single drain placed at the level of the diaphragm with its tip directed towards the apex. Port-site local anaesthetic infiltration was done, and the patient was reversed and extubated on the table. Intraoperatively, the patient was hemodynamically stable. However, desaturation up to $88 \%$ was noted, which was managed with $100 \%$ oxygen, and $3-\mathrm{cm} \mathrm{H}_{2} \mathrm{O}$ positive end-expiratory pressure; $\mathrm{SpO}_{2}$ was maintained within $92-94 \%$ after that. The postoperative course was uneventful; the chest drain was removed on postoperative day 3. Chest X-ray showed good lung expansion, and the child was symptom-free. Four weeks' follow-up was uneventful with good lung expansion (Figure 1). Histopathology for pleura was suggestive of nonspecific pleuritis. GeneXpert did not reveal Mycobacterium tuberculosis.

The present case shows the feasibility and safety of U-VATS for empyema in a 2-year-old child. However, the case had challenges from both anaesthesia and surgical viewpoints. Although double-lumen tube (DLT) is the gold standard for lung isolation, the appropriate size DLT for a 2-year-old child is not available, and the same is true of the univent tube. Further, an uncuffed tube has a risk of an improper seal, contrary to a cuffed tube, which has a risk of blocking the right upper lobe ventilation. Lung mechanics in infants and young children are also not favorable in the lateral position due to softer chest walls and relatively higher diaphragmatic positions [3, 4]. All these factors make

Address for correspondence: Dr. Habib Md Reazaul Karim, Department of Anaesthesiology and Critical Care, All India Institute of Medical Sciences, Raipur, India, phone: 91-9612372585, e-mail: drhabibkarim@gmail.com

Received: 16.03 .2021 , accepted: 16.05 .2021$. 

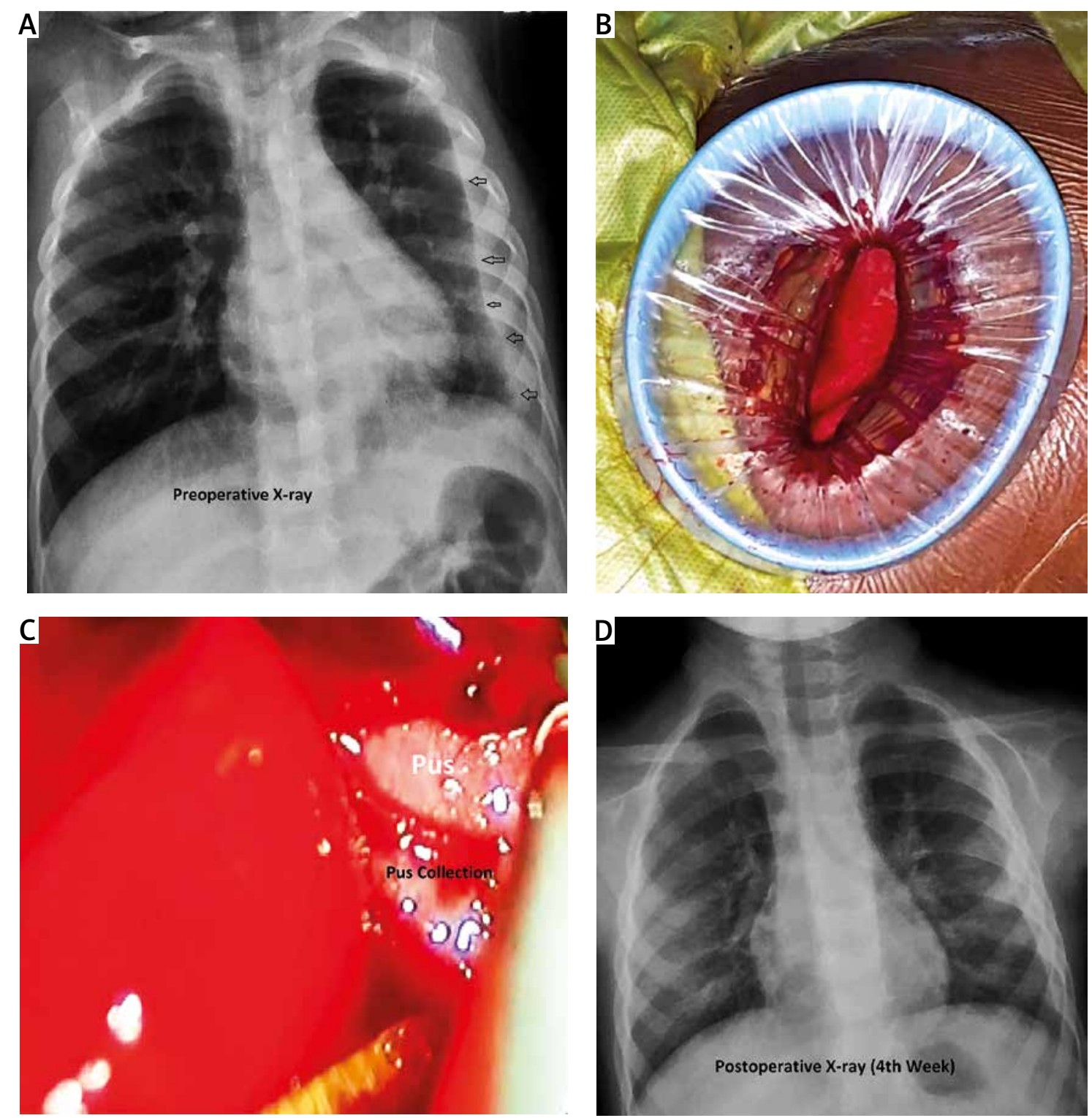

Figure 1. Preoperative chest X-ray showing loculated collection and left lung compression (A), surgical incision for U-VATS with wound protector in-situ (B), pus collection seen through endoscope (C), and expanded lung in postoperative chest X-ray (D)

OLV in young children challenging. Information on OLV for U-VATS in such young children is also scarce or missing.

The open thoracotomy leads to significant morbidity in a pediatric patient. Development of thoracic musculoskeletal and nervous structures during the first two years of life is crucial, and open thoracotomy carries the risk of developing scoliosis, winging of the scapula and muscular weakness. On the other hand, using traditional VATS bimanual instrumentation, nullifying the torsional angle is not possible [5]. U-VATS has a significant advantage regarding these aspects. Nevertheless, the learning curve is steep, and it is necessary to have good knowledge of the anatomical characteristics of the chest as the morphology of the pediatric chest changes with age [4]. Hence various morphological characteristics have to be acknowledged before an incision, and pre-incision ultrasound helped us delineate the diaphragmatic position.
U-VATS is an emerging technique, and knowledge, especially in the pediatric population, is minimal. It makes U-VATS in young children a challenging task. In 2004 Rocco et al. described this technique for diagnostic procedures and pneumothorax in adults [6]. Gonzalez-Rivas et al. in 2011 applied this technique for anatomical lung resection in adults, which bought a new evolution in the field of thoracic surgery [7]. Aragon et al. reported the first experience in U-VATS in an 11-year old male patient [8]. Later FernandezPineda et al., 2019, described their experience with modified U-VATS in 11 children with pulmonary nodules [9]. Recently, U-VATS has been used in various complex thoracic procedures in pediatric patients, primarily across advanced countries and set-ups with success. Recent literature shows a satisfactory result for U-VATS, even for stage III empyema in adult patients [10]. We believe that the successful and safe management of OLV using a single-lumen tube for 
U-VATS in a 2-year-old with chronic empyema stage III will further boost the use of this technique.

In conclusion, surgical treatment of stage-III empyema by U-VATS technique using OLV in a 2-year-old is a challenge but feasible and safe.

\section{Disclosure}

The authors report no conflict of interest.

\section{References}

1. Sakran W, Ababseh Zel D, Miron D, Koren A. Thoracic empyema in children: clinical presentation, microbiology analysis and therapeutic options. J Infect Chemother 2014; 20: 262-265.

2. Ismail M, Nachira D, Meacci E, Ferretti GM, Swierzy M, Englisch JP, Saidy RRO, Faber S, Congedo MT, Chiappetta M, Ciavarella LP, Margaritora S, Rueckert JC. Uniportal video-assisted thoracic surgery in the treatment of pleural empyema. J Thorac Dis 2018; 10 (Suppl 31): S3696-S3703.
3. Fabila TS, Menghraj SJ. One lung ventilation strategies for infants and children undergoing video assisted thoracoscopic surgery. Indian J Anaesth 2013; 57: 339-344.

4. García Asensio D, Fernández Martín M. Pitfalls and variants in pediatric chest imaging. Radiologia 2016; 58 Suppl 2: 58-69.

5. Casal BI, Miguez F, Gomez Tellado MA, Gonzalez RD. Thinking uniportal in pediatric thoracic surgery. Pediatric Med 2019; 2: 13.

6. Rocco G, Martin-Ucar A, Passera E. Uniportal VATS wedge pulmonary resections. Ann Thorac Surg 2004; 77: 726-728.

7. Gonzalez-Rivas D, Delgado M, Fieira E, Mendez L. Single-port video-assisted thoracoscopic lobectomy with pulmonary artery reconstruction. Interact Cardiovasc Thorac Surg 2013; 17: 889-891.

8. Aragón J, Méndez IP. First case report of single port video-assisted thoracoscopic middle lobectomy for the treatment of pulmonary aspergilloma in a pediatric patient. Eur J Pediatr Surg Rep 2013; 1: 12-14.

9. Fernandez-Pineda I, Seims AD, VanHouwelingen L, Abdelhafeez $H, W u H$, Wu J, Murphy AJ, Davidoff AM. Modified uniportal video-assisted thoracic surgery versus three-port approach for lung nodule biopsy in pediatric cancer patients. J Laparoendosc Adv Surg Tech A 2019; 29: 409-414.

10. van Middendorp LB, Franssen S, Gillissen S, Maessen JG, Hulsewé KWE, Vissers YLJ, de Loos ER. Uniportal video-assisted thoracoscopy is a safe approach in patients with empyema requiring surgery. J Thorac Dis 2020; 12: 1460-1466. 